\title{
Effects of Affected Side One Leg Standing Training with PNF Sprinter and Skater Patterns on the Balance and Gait Function in Hemiplegic Patients
}

\author{
IKHWAN EUN \\ Department of Rehabilitation Science, Graduate School of Daegu University, \\ Gyeongsangbuk-do, SOUTH KOREA \\ SEUNGMIN NAM* \\ Department of Physical Therapy, Yeungnam University Collage, \\ Daegu, SOUTH KOREA \\ *Corresponding author : SeungMin Nam
}

\begin{abstract}
This study was conducted to investigate the effect of affected side one leg standing training with PNF sprinter and skater patterns, which have a pattern like that of actual gait, on the balance function and gait function of chronic hemiplegic patients due to stroke. 23 inpatients diagnosed with stroke due to hemiplegia at a rehabilitation hospital located in South Korea. The patients were randomly divided into a experimental group and a control group. The experimental group performed PNF Sprinter and Skater Pattern training, and the control group performed balance pad training. Each group was trained for 30 minutes three times a week for six weeks. Static balance was measured using Biorescue, while dynamic balance was measured using the TUG. Gait function was measured using 10MWT, 6MWT. Both groups exhibited statistically significant improvements in static and dynamic balance function after the training $(\mathrm{p}<.05)$. The experimental group exhibited statistically significant increases in gait function after training $(\mathrm{p}<.05)$. A significant difference was observed between the two groups $(\mathrm{p}<.05)$. One leg standing training on the affected side using the PNF sprinter and skater patterns resulted in significant improvement in both balance and functional performance of gait in hemiplegic patients due to chronic stroke.
\end{abstract}

Key-Words: - Stroke, Hemiplegic patients, Sprinter and Skater Patterns, Static Balance Function, Dynamic Balance Function, Gait Function.

Received: April 15, 2021. Revised: September 26, 2021. Accepted: October 12, 2021. Published: October 29, 2021.

\section{Introduction}

Stroke is defined as a neurological defect caused by damage to blood vessel in the brain [1]. Stroke is one of the main causes of death, and in recent years, the age range of onset is gradually decreasing. Therefore, there is increasing interest in effective therapeutic interventions for stroke patients in clinical practice. Stroke generally suffers from spatiotemporal damage and various damage to cognition and motor function, such as aphasia, unilateral neglect, balance and gait disorders [2]. In particular, hemiplegia patients due to stroke cannot take a symmetric position and take an asymmetric position because their ability to support weight on the lower extremity of the affected side is poor. In addition, abnormal equilibrium reactions result in abnormal body sway, decreased weight support in the affected side, and increased risk of falling $[3,4]$.
In addition, due to the deterioration of the balance ability, a problem occurs in the gait ability and asymmetric position is induced during gait [5]. Due to the loss of these abilities, 20 to $30 \%$ of stroke patients lose their gait ability after acute, and most of the remaining patents also have gait disorder [6]. As such, the decrease in the balance and gait ability of hemiplegia patients has a negative effect on the daily activity of life [5].

Task-oriented training, visual feedback training, Bobath approach, and PNF are widely used as rehabilitation therapeutic approaches to improve balance and gait ability through enhancement of low extremity muscle strength of stroke patients [7]. However, in the case of gait, it is not achieved only by movement of lower extremity. Since the coordinative movement of the limb and the stability of the trunk are necessary, an intervention method that can practice the stability of the trunk and the 
connected movement of the limbs is necessary accordingly.

According to a recent study, sprinter and skater patterns were formulated as a coordination system between limbs without focusing only on low extremity movement by integrating each segmental pattern suggested by PNF. Therapy using this pattern is being performed to improve the balance and gait ability of hemiplegia patients [8]. Gait is not a movement that occurs only in a local area, but a movement that occurs mutually by combining several joints and muscles [9]. In other words, since gait is a movement that occurs in a standing position against gravity, it has been reported that gait training using PNF is better to train in a situation similar to the actual situation [10]. According to previous studies, it was reported that the intervention applying the sprinter and skater patterns is effective in strengthening low extremity muscle strength and enhancing static and dynamic balance [11]. In addition, it has been reported that it is effective in increasing gait speed and enhancing gait ability [12]. As such, sprinter and skater patterns are effective in improving the balance and gait ability of hemiplegia patients. However, most previous studies applied treatment by separating sprinter and skater patterns, and studies that applied sprinter and skater patterns at the same time are insufficient. In addition, studies on gait endurance after application of treatment were insufficient.

Therefore, this study was conducted to investigate the effect of affected side one leg standing training with PNF sprinter and skater patterns, which have a pattern like that of actual gait, on the balance function and gait function of chronic hemiplegic patients due to stroke.

\section{Methods}

\subsection{Subjects}

This study was conducted 30 patients who were hospitalized after being diagnosed with hemiplegia due to stroke at a rehabilitation hospital in D city in 2020. The contents of the experiment were explained to all subjects participating in the experiment, voluntary consent was sought, and it was conducted after obtaining approval (1040621201911-HR-030-02) by the Bioethics Committee. The intervention period was conducted for 6 weeks. 15 subjects in the experimental group using the PNF sprinter and skater pattern and 15 subjects in the control group using the balance pad were randomly assigned, and the study was conducted for a total of 6 weeks, 3 times a week for 30 minutes for each group. The criteria for selecting subjects for the study were those who were diagnosed as having a stroke and were more than 10 months after the onset, those who could understand the research content and communicate with them, and those who could stand one leg on the affected side to perform the task.

\subsection{Study protocol}

In both the experimental group and the control group, general rehabilitation treatment was performed 5 times a week for 6 weeks, 30 minutes each time. The general rehabilitation treatment was conservative exercise treatment, and functional exercise and muscle strength exercise were performed. In addition, additional training was performed three times a week for 30 minutes each time according to the target group. The experimental group applied PNF sprinter and skater patterns. The intervention of the onelegged standing training using the PNF sprinter and skater pattern was performed by a physical therapist for 30 minutes, 3 times a week, for 6 weeks. PNF sprinter and skater patterns were individually practiced in the first 10 minutes of the 30 minutes of intervention, and the PNF sprinter and skater patterns cooperative pattern exercise was followed in the latter 20 minutes. During the 6-week period, the difficulty was increased by increasing the number of times or by increasing the speed of posture conversion (fig 1). The control group performed squat training 10 times, 1 set total 5 times, and functional reach test 20 times, 1 set total 5 times. It was carried out with the non-affected side and the affected side, respectively (fig 2).

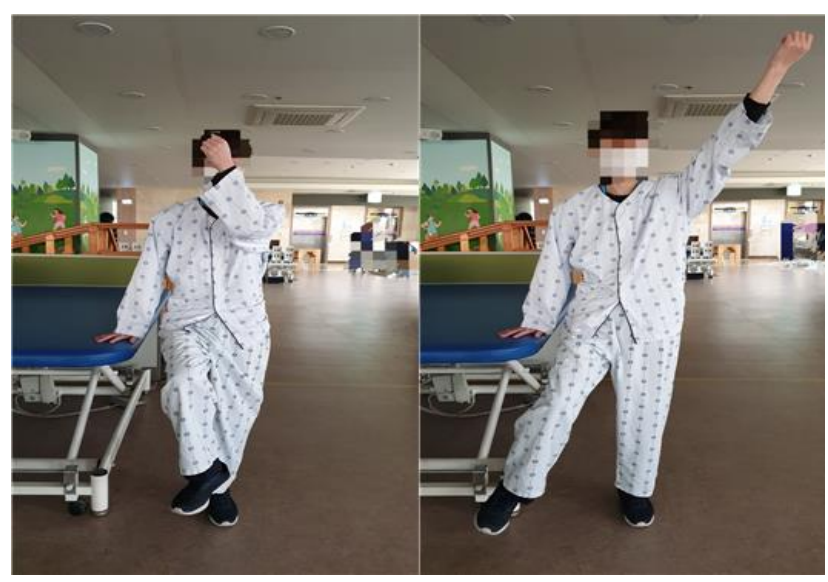

Fig. 1: Sprinter pattern \& Skater pattern 


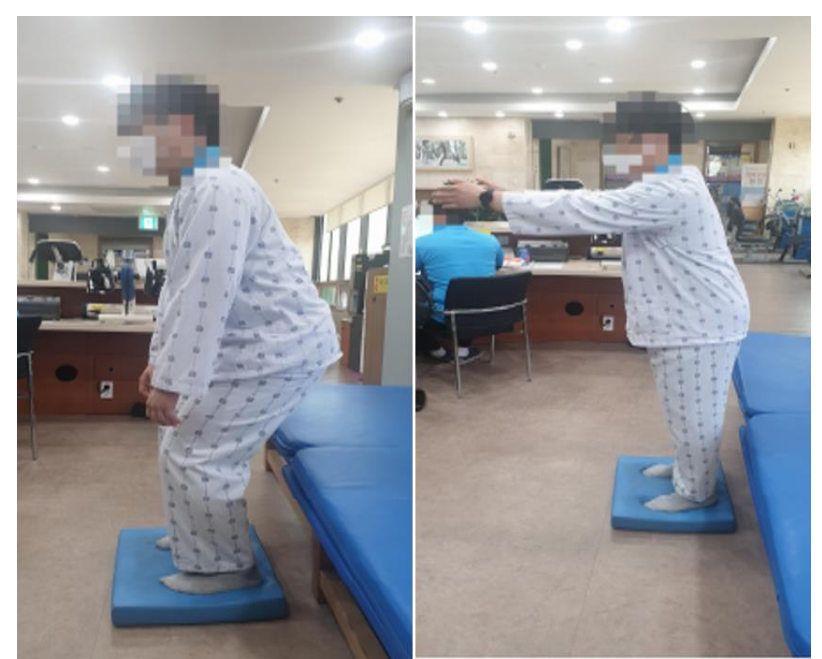

Fig. 2: Balance pad training

\subsection{Measurement Tools and Measurement Methods}

\subsubsection{Biorescue}

The Biorescue biofeedback analysis system (Biorescue, France) is a device that measures the balance ability of various subjects such as patients, the general public, and athletes. It is a tool that can measure length $(\mathrm{mm})$ and average speed $(\mathrm{cm} / \mathrm{s})$. In order to evaluate the static balance ability, the limit of stability at the standing posture was measured. Of the eight directions indicated by the monitor, the total distance and area of the center of gravity was measured for the weight movements before, after, left, and right. The device explained the measurement method through a monitor and first demonstrated it. The subject held a leg forward at $30^{\circ}$ in the standing position, centered in a forwardlooking position, and then used an ankle joint strategy to achieve maximum range without losing balance. The limit of their ability to move from their center of gravity was measured.

\subsubsection{Time Up \& Go Test (TUG)}

Time Up \& Go Test (TUG) was conducted to evaluate the dynamic balance ability of stroke patients. This test is to measure the average time by sitting down on a $46 \mathrm{~cm}$ highchair with armrests on a flat floor, then getting up and going back $3 \mathrm{~m}$ to return and sitting again three times.

\subsubsection{0-Meter Walk Test (10 MWT)}

The 10-meter walk test (10 MWT) is a test method that evaluates walking ability by measuring the walking speed of the examinee.19 No special equipment is required, so you can easily evaluate your walking ability in a short time. The method measures the time required to walk $10 \mathrm{~m}$ by taking into account acceleration and deceleration between the start and the end and measures a total of three times to obtain the average.

\subsubsection{Modified 6-Minute Walk Test (Modified 6MWT)}

The Modified 6-Minute Walk test is a test that can evaluate the muscular endurance and gait ability of a stroke patients. It measures the total distance walked while walking for 6 minutes. I had to make a round trip. The total distance traveled for 6 minutes was recorded in meters $(\mathrm{m})$. However, since it must be conducted indoors, it is difficult to prepare a space with a straight distance of $50 \mathrm{~m}$, and if the rotation is made 180 degrees at the turnaround point, there may be damage in the walking distance, so a separate track was produced and a $6 \mathrm{~m}$ walking test was conducted.

\subsection{Statistical analyses}

The Shapiro-Wilk test was performed to check for the normal distribution of each measurement item, and the results for all items satisfied normality. The data were presented as mean \pm standard deviation (Mean $\pm \mathrm{SD}$ ), and the general characteristics of the subjects were presented as descriptive statistics. There was no significant difference between each group. A paired t-test was performed to compare pre and post training performance within the Experimental group and control groups. An independent t-test was performed for between group comparisons. The data collected for this study was statistically processed using SPSS 23.0 for Windows (IBM, New York, USA) and the statistical significance level $\mathrm{P}$ value of $<0.05$.

\section{Results}

A homogeneity test showed no statistical difference between the groups ( $p>.05)$ (Table 1). The results of the LOS, TUG showed a significant increase after training in both the experimental group and the control group $(p<.05)$, with no significant difference between the groups ( $p>.05)$. The results of the 10MWT, 6MWT showed significant differences after training in the experimental group $(\mathrm{p}<.05)$ but not in the control group ( $p>.05)$. In these two tests, significant differences were observed between the groups $(\mathrm{p}<.05)($ Table 2$)$. 
Table 1. General characteristics of subjects

\begin{tabular}{ccccc}
\hline Group & $\begin{array}{c}\mathrm{EG} \\
(\mathrm{N}=11)\end{array}$ & $\begin{array}{c}\mathrm{CG} \\
(\mathrm{N}=12)\end{array}$ & $\mathrm{x}^{2} / \mathrm{t}$ & $p$ \\
\hline Gender (M/F) & $7 / 4$ & $4 / 8$ & 1.072 & .300 \\
Affected side (Lt/Rt) & $5 / 6$ & $9 / 3$ & 1.046 & .306 \\
Age (year) & $64.45 \pm 12.91$ & $66.08 \pm 10.37$ & -.335 & .741 \\
Height (cm) & $159.54 \pm 12.10$ & $159.58 \pm 9.41$ & -.008 & .993 \\
Weight (kg) & $61.45 \pm 7.04$ & $61.75 \pm 11.94$ & -.071 & .944 \\
On Set (month) & $24.90 \pm 23.08$ & $19.08 \pm 15.58$ & .715 & .482 \\
\hline
\end{tabular}

Mean \pm SD: Mean \pm Standard Deviation

EG: PNF Sprinter, Skater training group

CG: Traditional balance training group ${ }^{*} p<.05$

Table 2. Comparison of balance and gait function for each group

\begin{tabular}{|c|c|c|c|c|}
\hline & & $\begin{array}{c}\text { EG } \\
(\text { Mean } \pm \text { SD })\end{array}$ & $\begin{array}{c}\mathrm{CG} \\
(\mathrm{Mean} \pm \mathrm{SD})\end{array}$ & $p$ \\
\hline \multirow{3}{*}{ LOSF } & pre & $2107.63 \pm 1024.05$ & $1933.83 \pm 552.26$ & .613 \\
\hline & post & $4822.09 \pm 2751.64$ & $4039.50 \pm 890.14$ & .360 \\
\hline & $p$ & $.001^{*}$ & $.000^{*}$ & \\
\hline \multirow{3}{*}{ LOSB } & pre & $1337.27 \pm 789.56$ & $1150.91 \pm 407.72$ & .479 \\
\hline & post & $2180.63 \pm 1188.33$ & $2424.66 \pm 980.62$ & .596 \\
\hline & $p$ & $.002^{*}$ & $.000^{*}$ & \\
\hline \multirow{3}{*}{ LOSL } & pre & $1700.18 \pm 948.57$ & $1539.91 \pm 514.54$ & .615 \\
\hline & post & $3356.36 \pm 1857.91$ & $3348.91 \pm 864.37$ & .990 \\
\hline & $p$ & $.000^{*}$ & $.000^{*}$ & \\
\hline \multirow{3}{*}{ LOSR } & pre & $1783.18 \pm 851.59$ & $1526.91 \pm 505.74$ & .385 \\
\hline & post & $3611.45 \pm 2075.10$ & $3089.66 \pm 929.82$ & .438 \\
\hline & $p$ & $.001^{*}$ & $.000^{*}$ & \\
\hline \multirow{3}{*}{ TUG } & pre & $29.44 \pm 31.99$ & $21.25 \pm 9.20$ & .405 \\
\hline & post & $26.11 \pm 29.28$ & $18.32 \pm 8.24$ & .386 \\
\hline & $p$ & $.013^{*}$ & $.016^{*}$ & \\
\hline \multirow{3}{*}{ 10MWT } & pre & $15.61 \pm 4.07$ & $14.57 \pm 4.7$ & .582 \\
\hline & post & $11.26 \pm 2.70$ & $14.96 \pm 4.87$ & .037 \\
\hline & $p$ & $.000^{*}$ & .216 & \\
\hline
\end{tabular}




\begin{tabular}{lcccc}
\hline & pre & $240.21 \pm 81.74$ & $236.90 \pm 57.85$ & .911 \\
6MWT & post & $303.55 \pm 89.69$ & $237.08 \pm 59.35$ & $.047^{*}$ \\
& $p$ & $.001^{*}$ & .964 & \\
\hline
\end{tabular}

Mean \pm SD: Mean \pm Standard Deviation

EG: PNF Sprinter, Skater training group

CG: Traditional balance training group

LOSF: Limit of Stability Forward

LOSB: Limit of Stability Back

LOSL: Limit of Stability Left

LOSR: Limit of Stability Right

TUG: Time Up \& Go

10MWT: 10-Meter Walk Test

6MWT: 6-minute Walk Test

${ }^{*} p<.05$

\section{Discussion}

In this study, the sprinter and skater pattern training, which is an upper and lower extremity coordination exercise that systematizes the asymmetric inter-limb coordination system, which is one of the various forms of coordination, according to the characteristics of normal gait, is the static balance ability, dynamic balance ability, and gait ability of hemiplegic patients. Recently, many studies have been conducted to improve the gait of stroke patients. Various studies have been conducted, such as lower extremity muscle strengthening training, gait training using a treadmill, training using motion images, taskoriented exercise, and training using robots and virtual reality. However, since gait is the result of coordinated movement in which biomechanical and neuromuscular factors are combined, research on coordinated movement between the extremities is being conducted recently [13]. Therefore purpose of this study was to propose one of various methods of gait training that is helpful for balance and gait in hemiplegic patients due to stroke by examining its effect on.

Previous studies have focused on selective coordination training of the upper or lower limbs. In addition, there is a lack of research on coordination training between the upper and lower limbs using the entire upper and lower limbs, and there is a limitation in that there is only repetition of movements in a stable posture. In the case of walking, since the cooperative movement of the limbs using the entire limb and the stability of the trunk using the entire limb is required, an intervention method that can practice the stability

of the trunk and the connected movements of the limbs is necessary accordingly. In addition, since gait is a movement that occurs in a posture exposed to gravity, gait training using PNF is recommended to be trained in a situation like the actual situation. Therefore, this study performed gait training using cooperative movements between limbs, which are asymmetrical, using a PNF sprinter and skater pattern in a standing position.

As a result of this study, both the experimental group and the control group improved the static balance function and the dynamic balance function. However, there was no significant difference between the groups. Significant difference occurred before and after the intervention of the experimental group was that one leg standing training on the affected side without separate balance ability exercise increased the proprioceptive sensation of the ankle joint, thereby balancing itself before, after, and after. It is thought that the range of lateral movement has increased. In addition, the improvement of balance ability in this study induces symmetrical movements of the subject's torso, shoulders, and pelvis by the onelegged standing training on the affected side using the sprinter and skater patterns in this study $[14,15]$. It is also believed that this may be because the center of gravity biased on the non-affected side can be moved to the mid-line of the trunk more by inducing the activation of the affected side [16].

As a result of this study, there was a significant improvement in gait function in the experimental group. However, there was no significant improvement in the control group. There were significant differences between groups. These results show that the PNF sprinter and skater patterns learn not only the cross extension and 
flexion of both lower limbs in the same way as walking in a standing position, but also the cross and asymmetric rhythmic movements between the arms and limbs. It is thought that this is because by learning the mutual movements of the pelvis, the basic trunk and limb movements required for walking are more effectively learned [17]. In addition, when the body and limbs are connected by muscle chains when performing movements, the muscles of the trunk are also activated when force is transmitted from the arms to the legs around the trunk, and it is thought that not only static and dynamic balance but also walking ability is improved [18]. Also, it has been reported that the improvement of the weight transfer ability and balance to the affected side during the stance phase widens the stride [19]. In this study, balance ability increased after one leg standing training on the affected side using PNF sprinter and skater patterns, and walking speed was also significantly increased in the experimental group than in the control group. It was found that this one leg standing training to the affected side using PNF sprinter and skater patterns was effective in improving balance and gait ability of chronic hemiplegic patients. In addition, sprinter and skater movements that are easy to remember can be easily followed by providing audio-visual materials such as video data to patients, and through one-to-many group treatments rather than one-to-one treatments, it is possible to induce a sense of homogeneity or a sense of competition among subjects [18].

The limitations of this study are as follows. First, it is difficult to generalize to hemiplegic patients due to all strokes due to the small number of subjects for the study. Second, because the intervention period was short and the frequency was small, it was not possible to determine how long the actual effect lasted. Third, it has limitations in controlling various interventions, such as electrotherapy and occupational therapy, because it targets patients who are hospitalized and undergoing rehabilitation. This is likely to act as a factor influencing the research results.

\section{Conclusion}

In summarizing the results of this study, one leg standing training on the affected side using the PNF sprinter and skater patterns resulted in significant improvement in both balance and functional performance of gait in hemiplegic patients due to chronic stroke. Gait is a coordinated movement in which biomechanical and neuromuscular factors are combined. Therefore, one leg standing training to the affected side using PNF sprinter and skater patterns could be usefully used as an effective training method to improve balance and gait ability of hemiplegic patients due to chronic stroke.

\section{Acknowledgement:}

This work was supported by the National Research Foundation of Korea(NRF) grant funded by the Korea government(MSIT) (No. NRF2021R1G1A1092383).

\section{References:}

[1] Sampaio-Baptista, C., Sanders, ZB., JohansenBerg, H, Structural plasticity in adulthood with motor learning and stroke rehabilitation. Annu Rev Neurosci, Vol. 41, 2018, pp. 25-40.

[2] Zhang, JJ., Fong, KN., Welage, N., Liu, KP, The activation of the mirror neuron system during action observation and action execution with mirror visual feedback in stroke: a systematic review. Neural plasticity, 2018.

[3] Sinsurin, K., Srisangboriboon, S., Vachalathiti, $\mathrm{R}$, Side-to-side differences in lower extremity biomechanics during multi-directional jump landing in volleyball athletes. Eur J Sport Sci, Vol. 17, No. 6, 2017, pp. 699-709.

[4] Jeon, MJ., Moon, JH., Cho, HY, Effects of virtual reality combined with balance training on upper limb function, balance, and activities of daily living in persons with acute stroke: a preliminary study. Phys Ther Rehab Sci, Vol. 8, No. 4, 2019, pp. 187-193.

[5] Kim, EJ., Hwang, BY., Kim, JH, The effect of core strength exercises on balance and walking in patients with stroke. J Kor Phys Ther, Vol. 21, No. 4, 2009, pp. 17-22.

[6] Van De Port, IG., Kwakkel, G., Van Wijk, I., Lindeman, E, Susceptibility to deterioration of mobility long-term after stroke: a prospective cohort study. Stroke, Vol. 37, No.1, 2006, pp. 167-171.

[7] Langhorne, P., Bernhardt, J., Kwakkel, G, Stroke rehabilitation. The Lancet Vol. 377, No. 9778, 2011, pp. 1693-1702.

[8] Kim, JC., Lim, JH, The effects of coordinative locomotor training on coordination and gait in chronic stroke patients: a randomized controlled pilot trial. J Exerc Rehabil, Vol. 14, No. 6, 2018, pp. 1010.

[9] Krkeljas, Z, Changes in gait and posture as factors of dynamic stability during walking in pregnancy. Hum Mov Sci, Vol, 58, 2018, pp. 315-320.

[10] Lee, MK., Yun, TW., Kim, YH., Lim, JH, Effect of gait training using PNF on balance and walking ability in person with chronic 
stroke (Single Subject Design). PNF Mov, Vol. 10, No. 1, 2012, pp. 43-52.

[11] Lim, CG, The effects of proprioceptive neuromuscular facilitation (PNF) pattern exercise using the sprinter and the skater on balance and gait function in the stroke patients. J Kor Phys Ther, Vol. 26, No. 4, 2014, pp. 249-256.

[12] Kim, K., Lee, DK., Jung, SI, Effect of coordination movement using the PNF pattern underwater on the balance and gait of stroke patients. J Phys Ther Sci, Vol. 27, No. 12, 2015, pp. 3699-3701.

[13] Morone, G., Paolucci, S., Cherubini, A., De Angelis, D., Venturiero, V., Coiro, P., Iosa, M, Robot-assisted gait training for stroke patients: current state of the art and perspectives of robotics. Neuropsychiatr Dis Treat, Vol. 13, 2017, pp. 1303.

[14] Kim, EK., Lee, DK., Kim, YM, Effects of aquatic PNF lower extremity patterns on balance and ADL of stroke patients. J Phys Ther Sci, Vol. 27, No. 1, 2015, pp. 213-215.

[15] Liang, JN., Ho, KY., Hung, V., Reilly, A., Wood, R., Yuskov, N., Lee, YJ, Effects of augmented somatosensory input using vibratory insoles to improve walking in individuals with chronic post-stroke hemiparesis. Gait \& Posture, Vol. 86, 2021, pp. 77-82.

[16] Jung, K., Kim, Y., Chung, Y., Hwang, S, Weight-shift training improves trunk control, proprioception, and balance in patients with chronic hemiparetic stroke. Tohoku J Exp Med, Vol. 232, No. 3, 2014, pp. 195-199.

[17] Jeong, WS., Park, SK., Park, JH., Lee, HG., Kim, KY, Effect of PNF combination patterns on muscle activity of the lower extremities and gait ability in stroke patients. J Kor Cont Associ, Vol. 12, No. 1, 2012, pp. 318-328.

[18] Lim, JH., Lee, MK., Kim, TY., Ko, HE, The combination of PNF patterns for coordinative locomotor training. PNF Mov, Vol. 11, No. 1, 2013, pp.17-25.

[19] Wu, YT., Choe, YW., Peng, C., Kim, MK, The immediate effects of posterior pelvic tilt with taping on pelvic inclination, gait function and balance in chronic stroke patients. $\mathrm{J}$ Kor Soc Phys Med, Vol. 12, No, 3, 2017, pp. 1121.
Contribution of Individual Authors to the Creation of a Scientific Article (Ghostwriting Policy)

IkHwan Eun has organized and executed the experiments.

SeungMin Nam was responsible for the Statistics.

\section{Creative Commons Attribution License 4.0}

(Attribution 4.0 International, CC BY 4.0)

This article is published under the terms of the Creative Commons Attribution License 4.0 https://creativecommons.org/licenses/by/4.0/deed.e $\underline{\mathrm{n} \text { US }}$ 